\title{
Risk factors for postoperative complications after elective ileocolic resection for Crohn's disease: a retrospective study
}

\author{
Antonios Gklavas, Aikaterini Poulaki, Dionysios Dellaportas, loannis Papaconstantinou
}

Aretaieion University Hospital, National and Kapodistrian University of Athens, Athens, Greece

Abstract

Background The incidence of postoperative complications (POC) in patients with Crohn's disease (CD) who undergo intestinal resection is high. The literature provides conflicting data about the risk factors for POC, especially regarding preoperative immunosuppressive medications. The purpose of this study was to evaluate the impact of anti-tumor necrosis factor (TNF) agents on the postoperative course and identify other predictors for POC after ileocolic resection (ICR).

Methods This was a single-center retrospective study that included $153 \mathrm{CD}$ patients who underwent elective ICR in a Greek tertiary center between January 2010 and December 2018. Risk factors for overall POC and intra-abdominal septic complications (IASC) were assessed with univariate and multivariate analyses.

Results Overall POC and IASC occurred in 35 (22.9\%) and 19 (12.4\%) patients, respectively. In multivariate analysis, anti-TNF agents $(n=61)$, as either monotherapy or combination treatment, were not associated with an increased risk for overall POC (21.3\% vs. $23.9 \%, \mathrm{P}=0.71)$ or IASC ( $13.1 \%$ vs. $12.0 \%, \mathrm{P}=0.83$ ). Similarly, no combined immunosuppressive regimen significantly correlated with POC. Patients with perianal disease, disease duration $>10$ years, or previous intestinal resections had significantly higher rates of both overall POC and IASC. In multivariate analysis, previous resection was the only independent risk factor for overall POC (odds ratio [OR] 3.90, 95\% confidence interval [CI] 1.38-11.06; $\mathrm{P}=0.010$ ) and IASC (OR 4.56, 95\%CI 1.51-13.77; $\mathrm{P}=0.007)$.

Conclusions Preoperative administration of anti-TNF agents or other immunosuppressive regimens was not a risk factor for total POC or IASC. A history of previous resection independently correlated with both overall POC and IASC.

Keywords Crohn's disease, postoperative complications, intra-abdominal septic complications, anti-TNF, previous resections

Ann Gastroenterol 2020; 33 (6): 1-11

\section{Introduction}

Crohn's disease $(\mathrm{CD})$ is a chronic inflammatory condition that follows a course of alternating periods of relapse and remission and can affect any part of the gastrointestinal tract [1]. In 1998, the approval of infliximab, the first monoclonal antibody against tumor necrosis factor (TNF)- $\alpha$, changed the

$2^{\text {nd }}$ Surgical Department, Aretaieion University Hospital, National and Kapodistrian University of Athens, Athens, Greece

Conflict of Interest: None

Correspondence to: Antonios Gklavas, MD, $2^{\text {nd }}$ Department of Surgery, Aretaieion University Hospital, Vasilissis Sofias 76, 11528, Athens, Greece, e-mail: agklavas@gmail.com

Received 3 May 2020; accepted 29 June 2020; published online 16 September 2020

DOI: https://doi.org/10.20524/aog.2020.0533 modern conservative treatment of inflammatory bowel diseases (IBD) [2]. Despite immense progress in the era of biologic agents regarding the medical treatment of $\mathrm{CD}$, the need for intestinal resection remains high: at 1, 5 and 10 years after diagnosis the risk for intestinal surgery is $16 \%, 33 \%$ and $47 \%$, respectively [3].

Current evidence suggests high rates for postoperative complications (POC); up to $37 \%$ of $\mathrm{CD}$ patients who undergo surgery will experience POC [4]. Thus, several studies have tried to highlight the risk factors related to POC and research has been mainly focused on the preoperative immunosuppressive medication as a potential predictor of POC. However, the majority of current data regarding the association between medication and POC are extracted from observational, retrospective studies with high heterogeneity regarding POC classification (overall, anastomotic-related, septic vs. non-septic, minor vs. major), medication dosage, interval between last administration and surgery, and the examination of confounding factors such as nutritional status 
or coadministration of other immunosuppressive regimens [5]. Another confounding factor that should be taken into account is surgery in the emergency/urgent setting. These patients cannot be properly optimized preoperatively in order to minimize the risk for postoperative morbidity [6].

While the findings for certain categories of medication, such as steroids or immunomodulators (IM), are highly consistent in the literature $[7,8]$, data regarding biologic agents remain conflicting [9]. The purpose of this study was to evaluate the impact of anti-TNF therapy on the postoperative course and to identify other risk factors for overall and intra-abdominal septic POC in CD patients who undergo elective ileocolic resection (ICR).

\section{Patients and methods}

\section{Patient selection}

This was a single-center retrospective study based on a prospectively updated database of $218 \mathrm{CD}$ patients who underwent ICR with primary anastomosis in a tertiary referral center between January 2010 and December 2018. Exclusion criteria were age $<18$ years, defunction of the anastomosis with a proximal diverting ileostomy, emergency or urgent surgery, and missing or invalid data precluding analysis. Patients who simultaneously had small bowel resection or segmental colectomy or strictureplasty, apart from ICR, were included in the cohort. After these exclusion criteria were applied, 153 patients were eligible for analysis. This study was approved by the hospital's research and ethics committee.

\section{Preoperative data and management}

Data were retrieved using either the prospectively updated electronical IBD database or the patients' hospital medical records. Demographic variables included age, sex, smoking habit, body mass index (BMI) according to the World Health Organization (WHO) classification [10], and the American Society of Anesthesiologists (ASA) physical status score [11]. Disease phenotype was recorded according to the Montreal classification for CD [12]. Further disease characteristics included disease duration, previous intestinal resections, and the presence of extraintestinal manifestations. Preoperative serum albumin $(\mathrm{g} / \mathrm{dL})$ and hemoglobin $(\mathrm{g} / \mathrm{dL})$ were also recorded; the cut-off value of $3.0 \mathrm{~g} / \mathrm{dL}$ was used to discriminate low albumin concentration [13], while the severity of anemia was categorized according to WHO classification [14]. Intraoperative data included surgical approach (open vs. laparoscopic), concomitant intestinal resection or strictureplasty, and anastomotic configuration.

The administration of 5-aminosalicylates, IM (azathioprine, 5-mercaptopurine, methotrexate), and steroids in the timeframe of 4 weeks before surgery was recorded; there is inconsistency in the literature regarding the interval between last dose and surgery, ranging from 4-12 weeks, in order for a patient to be considered exposed to immunosuppressive treatment [15-17]. For the purposes of the present analysis, patients who received anti-TNF or other biologic agents within 3 months before resection were considered positive; this is in accordance with many previous studies $[15,16]$. Furthermore, the therapeutic regimens were classified according to whether patients were under monotherapy or combination treatment ( $\geq 2$ categories of medications).

Preoperative optimization included packed red blood cell transfusions, correction of nutritional deficiencies and fluid and electrolyte abnormalities, when appropriate. Weaning-off steroids was aimed at, when possible. The main strategy was to discontinue biological therapy at least 4 weeks before surgery.

\section{Surgical technique}

The majority of patients had undergone a thorough preoperative gastrointestinal mapping with ileocolonoscopy and computed tomography or magnetic resonance enterography. All operations were performed by the same specialist colorectal surgeon. ICRs were performed using an open or laparoscopic approach, according to the patient's disease characteristics; laparoscopic surgery was preferred for single stenotic disease of the ileocecal region. Patients with penetrating disease and abscesses or complex fistulas, need for multiple bowel resections, previous laparotomies or inability to tolerate pneumoperitoneum due to comorbidities, were operated using an open approach. Regardless of the surgical approach, the resection included only the macroscopically diseased bowel segment, whereas the mesentery was dissected and ligated close to the intestinal wall. The anastomotic configuration was selected according to the intraoperative findings: an end-toend, single layer, hand-sewn anastomosis (EEA) was performed for bowel edges of similar diameter; otherwise, a double layer, hand-sewn, side-to-side hand-sewn anastomosis (SSA) was preferred. The decision regarding concomitant intestinal resection was made according to preoperative evaluation and intraoperative findings. Strictureplasties were performed only for small bowel strictures. Chronic, high-dose steroid therapy, as well as severe intra-abdominal sepsis and malnutrition, were considered indications for anastomotic diversion.

\section{POC}

Early POC and mortality within 30 days after surgery were recorded. Intra-abdominal septic complications (IASC) included anastomotic leakage, intra-abdominal abscess and enterocutaneous fistula. Overall POC included, apart from IASC, postoperative ileus, urinary tract infection (UTI), respiratory tract infection (RTI), hemorrhage, wound infection, and unexplained fever. The aforementioned POC were documented by appropriate laboratory tests and/or imaging modalities. Lastly, the incidence of re-operation within 30 days was calculated. We evaluated possible risk factors for IASC and overall POC. 


\section{Statistical analysis}

Categorical variables were represented as absolute and relative frequencies (\%). Normality of distribution for quantitative variables was evaluated using the KolmogorovSmirnov and Shapiro-Wilk tests. Mean value ( \pm standard deviation) was used to describe normally distributed variables, whereas non-normally distributed ones were presented as median (25th-75th interquartile range [IQR]). Univariate analysis was conducted using Pearson's chi-square test or Fisher's exact test to compare categorical variables, where appropriate. When statistical significance was reached in a $2 \times 3$ contingency table, a post hoc analysis was conducted using the Bonferroni adjustment for P-value. The independent samples $t$-test was used for the comparison of normally distributed continuous data, whereas the Mann-Whitney $U$ test was used for non-parametrical variables. Multivariate analysis was conducted using a multiple logistic regression model for the calculation of odds ratio (OR) with $95 \%$ confidence interval (CI). All P-values were 2 -sided and values $<0.05$ were considered statistically significant. All calculations were performed using SPSS version 23.0 software (SPSS, Chicago, IL).

\section{Results}

\section{Baseline characteristics}

The demographic, clinical and surgical characteristics of 153 patients are presented in Table 1. Our population had a median age of 36 years (IQR 28-47 years), and included 86 male patients (56.2\%) and 61 active smokers (39.9\%). Single ICR was performed in most cases $(86.9 \%)$, whereas 20 patients underwent concomitant resection and/or strictureplasty. A laparoscopic approach was preferred in 19 patients (12.4\%), whereas EEA was the most common anastomotic configuration $(\mathrm{n}=128,83.7 \%)$. Severe anemia (hemoglobin $[\mathrm{Hb}]<8 \mathrm{~g} / \mathrm{dL}$ ) was not recorded in any patient and the majority of patients (86.5\%) had serum albumin $>3.0 \mathrm{~g} / \mathrm{dL}$. According to the ASA score, the study population had no severe comorbidities (ASA I/II, 93.5\%). Most patients had no perianal involvement (77.1\%) or previous resections (84.3\%).

Preoperative medical treatment was described according to the kind of medication and therapeutic regimen; anti-TNF was the most commonly used category (39.9\%), as either monotherapy $(25.5 \%)$ or combination treatment (14.4\%). Vedolizumab (3.3\%) and ustekinumab (2.0\%) were used only as monotherapy regimens. Steroid therapy was taken by $23.5 \%$ of patients. Homogeneity among patients who were under anti-TNF therapy or not was analyzed (Table 2). Statistically significant differences between the 2 groups were observed for median age ( 34 vs. 38.5 years, $\mathrm{P}=0.016$ ), age at diagnosis according to Montreal classification ( $\mathrm{P}=0.010)$, and steroid use, which was less frequent in anti-TNF patients (13.1\% vs. $30.4 \%$, $\mathrm{P}=0.013)$.
Table 1 Overall baseline characteristics $(n=153)$

\begin{tabular}{|c|c|}
\hline Characteristics & Value \\
\hline Age (years), median (IQR) & $36(28-47)$ \\
\hline Male sex, n (\%) & $86(56.2)$ \\
\hline Smoking, n (\%) & $61(39.9)$ \\
\hline $\begin{array}{l}\text { BMI }\left(\mathrm{kg} / \mathrm{m}^{2}\right) \text {, mean } \pm \mathrm{SD} \\
\quad<18.5, \mathrm{n}(\%) \\
18-25, \mathrm{n}(\%) \\
25-30, \mathrm{n}(\%) \\
30-35, \mathrm{n}(\%) \\
>35, \mathrm{n}(\%)\end{array}$ & $\begin{array}{l}22.9 \pm 3.9 \\
16(10.9) \\
89(60.5) \\
37(25.2) \\
4(2.7) \\
1(0.7)\end{array}$ \\
\hline $\begin{array}{l}\text { Hemoglobin }(\mathrm{g} / \mathrm{dL}) \text {, mean } \pm \mathrm{SD} \\
\text { No anemia, } \mathrm{n}(\%)^{\varsigma} \\
\text { Mild anemia, } \mathrm{n}(\%) \\
\text { Moderate anemia, } \mathrm{n}(\%) \\
\text { Severe anemia, } \mathrm{n}(\%)\end{array}$ & $\begin{array}{c}12.7 \pm 1.8 \\
65(62.5) \\
17(16.3) \\
22(21.2) \\
0(0)\end{array}$ \\
\hline $\begin{array}{l}\text { Albumin }(\mathrm{g} / \mathrm{dL}) \text {, median }(\mathrm{IQR}) \\
>3.0, \mathrm{n}(\%)\end{array}$ & $\begin{array}{l}3.7(3.3-4.1) \\
90(86.5)\end{array}$ \\
\hline ASA Score I/II, n (\%) & $143(93.5)$ \\
\hline $\begin{array}{l}\text { Montreal - Age, n (\%) } \\
\text { A1, } \\
\text { A2 } \\
\text { A3 }\end{array}$ & $\begin{array}{c}23(15.0) \\
101(66.0) \\
29(19)\end{array}$ \\
\hline $\begin{array}{l}\text { Montreal - Location, n (\%) } \\
\text { L1 } \\
\text { L2 } \\
\text { L3 }\end{array}$ & $\begin{array}{c}92(60.1) \\
9(5.9) \\
52(34.0)\end{array}$ \\
\hline Montreal - L4, n (\%) & $4(2.6)$ \\
\hline $\begin{array}{l}\text { Montreal - Behavior, n (\%) } \\
\text { B1 } \\
\text { B2 } \\
\text { B3 }\end{array}$ & $\begin{array}{c}9(5.9) \\
95(62.1) \\
49(32.0)\end{array}$ \\
\hline Perianal disease, $\mathrm{n}(\%)$ & $35(22.9)$ \\
\hline $\begin{array}{l}\text { Disease duration (years), median } \\
\text { (IQR) } \\
<5, \mathrm{n}(\%) \\
5-10, \mathrm{n}(\%) \\
>10, \mathrm{n}(\%)\end{array}$ & $\begin{array}{l}7.2(2.5-14.0) \\
62(40.5) \\
36(23.5) \\
55(35.9)\end{array}$ \\
\hline Extraintestinal manifestations, $\mathrm{n}(\%)$ & $48(31.4)$ \\
\hline Previous resection, $\mathrm{n}(\%)$ & $24(15.7)$ \\
\hline Laparoscopic approach, n (\%) & $19(12.4)$ \\
\hline $\begin{array}{l}\text { Procedure, } \mathrm{n}(\%) \\
\text { ICR } \\
\text { ICR + Strictureplasty } \\
\text { ICR + resection } \\
\text { ICR + resection + strictureplasty }\end{array}$ & $\begin{array}{c}133(86.9) \\
5(3.3) \\
13(8.5) \\
2(1.3)\end{array}$ \\
\hline $\begin{array}{l}\text { Anastomotic configuration, } \mathrm{n}(\%) \\
\text { EEA } \\
\text { SSA }\end{array}$ & $\begin{array}{c}128(83.7) \\
25(16.3)\end{array}$ \\
\hline $\begin{array}{l}\text { Medication, n (\%) } \\
\text { 5-ASA } \\
\text { IM } \\
\text { Steroids } \\
\text { Anti-TNF } \\
\text { Vedolizumab } \\
\text { Ustekinumab }\end{array}$ & $\begin{array}{c}12(7.8) \\
35(22.9) \\
36(23.5) \\
61(39.9) \\
5(3.3) \\
3(2.0)\end{array}$ \\
\hline
\end{tabular}

(Contd...) 
Table 1 (Continued)

\begin{tabular}{lc}
\hline Characteristics & Value \\
\hline Therapeutic regimen, $\mathrm{n}(\%)$ & \\
None & $44(28.8)$ \\
Monotherapy & $12(7.8)$ \\
IM & $19(12.4)$ \\
Steroid & $39(25.5)$ \\
Anti-TNF & $5(3.3)$ \\
Vedolizumab & $3(2.0)$ \\
Ustekinumab & \\
Combination therapy & $9(5.9)$ \\
Steroid + IM & $14(9.2)$ \\
Anti-TNF + IM & $8(5.2)$ \\
Anti-TNF + Steroids & \\
\hline
\end{tabular}

${ }^{5}$ World Health Organization classification; mild anemia when serum hemoglobin $(\mathrm{Hb}) 11-12 \mathrm{~g} / \mathrm{dL}$ for females and 11-13 g/dL for males, moderate for $\mathrm{Hb} 8-11 \mathrm{~g} / \mathrm{dL}$ and severe for $\mathrm{Hb}<8 \mathrm{~g} / \mathrm{dL}$ (16)

$I Q R$, interquartile range; $B M I$, body mass index; ASA, American Society of Anesthesiologists; ICR, ileocolic resection; EEA, end-to-end anastomosis; SSA, side-to-side anastomosis; 5-ASA, 5-aminosalicylic acid; TNF, tumor necrosis factor; IM, immunomodulators

\section{POC}

Overall, 35 patients (22.9\%) experienced POC (Table 3). Anastomotic leakage was the most common POC $(n=18,11.8 \%)$ and IASC was recorded in 19 patients (12.4\%). Postoperative ileus was the most frequent non-IASC complication (3.9\%), while postoperative hemorrhage and unexplained fever were recorded in 4 patients (2.6\%). Non-IASC septic complications included wound infections (2.0\%), UTI (1.3\%), and RTI (0.7\%). In total, 22 patients required reintervention (14.4\%); 17 with IASC (2 cases with anastomotic leak were treated conservatively), 4 with hemorrhage and 1 with postoperative bowel obstruction. No patient died during the 30-day postoperative period.

\section{Univariate analysis}

The analysis of preoperative medications showed no statistically significant differences in the rates of overall POC and IASC (Fig. 1). Steroid therapy was associated with a higher incidence of overall POC (33.3\%), although statistical significance was not reached $(\mathrm{P}=0.09)$. Patients with preoperative administration of anti-TNF agents had a nonsignificantly lower percentage of overall POC (21.3\% vs. $23.9 \%$, $\mathrm{P}=0.71)$ and a non-significantly higher percentage of IASC ( $13.1 \%$ vs. $12.0 \%, \mathrm{P}=0.83$ ). Similarly, the analysis according to the therapeutic regimen revealed no significant differences in overall and IASC rates (Fig. 2). Anti-TNF treatment was not associated with overall POC or IASC, either as monotherapy or in combination regimens. The administration of steroids, either as monotherapy or as a combination treatment with IM or anti-TNF, showed the highest incidences of overall POC, although the differences were statistically non-significant (31.6\%, $\mathrm{P}=0.35 ; 33.3 \%, \mathrm{P}=0.41 ; 37.5 \%, \mathrm{P}=0.37$, respectively). As for IASC, combined steroid and anti-TNF therapy had the highest rate $(25.0 \%, \mathrm{P}=0.23)$. Patients under a combined
anti-TNF and IM regimen showed insignificantly lower percentages of complications $(7.1 \%, \mathrm{P}>0.99)$. The number of patients receiving vedolizumab $(n=5)$ or ustekinumab $(n=3)$ was too small for analysis.

Demographic, clinical and surgical variables were used in the univariate analysis (Table 4). Three variables were associated with significantly higher rates of overall POC and IASC: perianal disease $(37.1 \%, \mathrm{P}=0.022$ and $25.7 \%, \mathrm{P}=0.016$, respectively), a history of previous intestinal resection (54.2\%, $\mathrm{P}<0.001$ and $37.5 \%, \mathrm{P}<0.001$, respectively), and disease duration ( $\mathrm{P}=0.012$ and 0.029 , respectively). Statistical significance for the "disease duration" variable was noted after the post hoc analysis for patients with $>10$ years of diagnosed CD compared to those with $<5$ years ( $36.4 \%$ vs. $16.1 \%, \mathrm{P}=0.012$ for overall POC and $21.8 \%$ vs. $6.5 \%, \mathrm{P}=0.016$ for IASC).

\section{Multivariate analysis}

Preoperative medication variables and others with a P-value $<0.25$ in the univariate analysis were inserted into a binary logistic regression model (Table 5). Previous resections were the only significant independent predictor for overall POC and IASC: OR 3.90, 95\%CI 1.38-11.06; $\mathrm{P}=0.010$, and $\mathrm{OR}$ 4.56, 95\% CI 1.51-13.77; $\mathrm{P}=0.007$, respectively. In contrast to the univariate analysis, perianal disease and duration of $\mathrm{CD}$ $>10$ years were not independently associated with a higher OR for POC. Preoperative medication was also non-significantly correlated with POC.

\section{Discussion}

In this retrospective study we tried to detect and analyze risk factors for POC in a Greek cohort of CD patients operated in a high-volume tertiary center, paying special attention to the patients' preoperative immunosuppressive therapy. In total, $22.9 \%$ of our patients experienced some kind of POC; this finding is comparable with complication rates reported in other studies $[15,18,19]$. Anastomosis-related complications, usually presenting as free peritoneal leakage or intraabdominal abscess leading to intra-abdominal sepsis, represent the category of POC causing most concern among colorectalIBD surgeons. The recognition of patients at a higher risk for IASC is crucial and affects the surgical strategy; anastomotic defunction with a temporary stoma is the preferred option for these patients. In our series the incidence of IASC was $12.4 \%$, also comparable with the current literature [17,20,21].

According to this study's findings, anti-TNF treatment within 3 months before surgery was not associated with a higher risk for overall POC or IASC. The great concern regarding the safety of anti-TNF agents is that their immense immunosuppressive effect could not only affect the patient's defense against infections, but theoretically impair the healing cascade as well, leading to septic and anastomotic complications. Clinical data from a large number of non-randomized, mainly 
Table 2 Comparison of baseline characteristic between patients under anti-TNF therapy and not

\begin{tabular}{|c|c|c|c|}
\hline Characteristics & Anti-TNF $(\mathrm{n}=61)$ & No Anti-TNF ( $\mathrm{n}=92)$ & P-value \\
\hline Age (years), median (IQR) & $34(25-41.5)$ & $38.5(31-49)$ & 0.016 \\
\hline Male sex, n (\%) & $33(54.1)$ & $53(57.6)$ & 0.67 \\
\hline Smoking, n (\%) & $20(32.8)$ & $41(44.6)$ & 0.15 \\
\hline $\mathrm{BMI}\left(\mathrm{kg} / \mathrm{m}^{2}\right)$, mean $\pm \mathrm{SD}$ & $22.7 \pm 4.3$ & $23.1 \pm 3.7$ & 0.54 \\
\hline $\begin{array}{l}<18.5, \mathrm{n}(\%) \\
18.5-25, \mathrm{n}(\%) \\
25-30, \mathrm{n}(\%) \\
30-35, \mathrm{n}(\%) \\
>35, \mathrm{n}(\%)\end{array}$ & $\begin{array}{c}8(13.6) \\
35(59.3) \\
12(20.3) \\
4(6.8) \\
0(0.0)\end{array}$ & $\begin{aligned} 8 & (9.1) \\
54 & (61.4) \\
25 & (28.4) \\
0 & (0.0) \\
1 & (1.1)\end{aligned}$ & 0.08 \\
\hline Hemoglobin $(\mathrm{g} / \mathrm{dL})$, mean $\pm \mathrm{SD}$ & $12.5 \pm 1.8$ & $12.9 \pm 1.9$ & 0.31 \\
\hline $\begin{array}{l}\text { No anemia, } \mathrm{n}(\%)^{\varsigma} \\
\text { Mild anemia, } \mathrm{n}(\%) \\
\text { Moderate anemia, } \mathrm{n}(\%) \\
\text { Severe anemia, } \mathrm{n}(\%)\end{array}$ & $\begin{array}{c}25(59.5) \\
7(16.7) \\
10(23.8) \\
0(0.0)\end{array}$ & $\begin{array}{c}40(64.5) \\
10(16.1) \\
12(19.4) \\
0(0.0)\end{array}$ & 0.84 \\
\hline $\begin{array}{l}\text { Albumin (g/dL), median (IQR) } \\
<3.0, \mathrm{n}(\%)\end{array}$ & $\begin{array}{l}3.7(3.3-4.1) \\
\quad 7(16.7)\end{array}$ & $\begin{array}{l}3.7(3.4-4.0) \\
\quad 7(11.3)\end{array}$ & $\begin{array}{l}0.67 \\
0.43\end{array}$ \\
\hline ASA Score I/II, n (\%) & $57(93.4)$ & $86(93.5)$ & $>0.99$ \\
\hline $\begin{array}{l}\text { Montreal - Age, n (\%) } \\
\text { A1 } \\
\text { A2 } \\
\text { A3 }\end{array}$ & $\begin{array}{c}15(24.6) \\
39(63.9) \\
7(11.5)\end{array}$ & $\begin{array}{c}8(8.7) \\
62(67.4) \\
22(23.9)\end{array}$ & 0.010 \\
\hline $\begin{array}{l}\text { Montreal - Location, n (\%) } \\
\text { L1 } \\
\text { L2 } \\
\text { L3 }\end{array}$ & $\begin{aligned} 32 & (52.5) \\
4 & (6.6) \\
25 & (41.0)\end{aligned}$ & $\begin{aligned} 60 & (65.2) \\
5 & (5.4) \\
27 & (29.3)\end{aligned}$ & 0.28 \\
\hline Montreal - L4, n (\%) & $2(3.3)$ & $2(2.2)$ & $>0.99$ \\
\hline $\begin{array}{l}\text { Montreal - Behavior, } \mathrm{n}(\%) \\
\text { B1 } \\
\text { B2 } \\
\text { B3 }\end{array}$ & $\begin{array}{c}3(4.9) \\
40(65.6) \\
18(29.5)\end{array}$ & $\begin{array}{c}6(6.5) \\
55(59.8) \\
31(33.7)\end{array}$ & 0.76 \\
\hline Perianal disease, $\mathrm{n}(\%)$ & $18(29.5)$ & $17(18.5)$ & 0.11 \\
\hline Disease duration (years), median (IQR) & $8.8(3.0-15.5)$ & $6.15(2.0-12.0)$ & 0.08 \\
\hline $\begin{array}{l}<5, \mathrm{n}(\%) \\
5-10, \mathrm{n}(\%) \\
>10, \mathrm{n}(\%)\end{array}$ & $\begin{array}{l}21(34.4) \\
14(23.0) \\
26(42.6)\end{array}$ & $\begin{array}{l}41(44.6) \\
22(23.9) \\
29(31.5)\end{array}$ & 0.33 \\
\hline Extraintestinal manifestations, $\mathrm{n}(\%)$ & $19(31.1)$ & $29(31.5)$ & 0.96 \\
\hline Previous resection, $\mathrm{n}(\%)$ & $13(21.3)$ & $11(12.0)$ & 0.12 \\
\hline Laparoscopic approach, n (\%) & $8(13.1)$ & $11(12.0)$ & 0.83 \\
\hline $\begin{array}{l}\text { Procedure, } \mathrm{n}(\%) \\
\text { ICR } \\
\text { ICR + Strictureplasty } \\
\text { ICR + resection } \\
\text { ICR + resection + strictureplasty }\end{array}$ & $\begin{array}{c}52(82.0) \\
3(4.9) \\
7(11.5) \\
1(1.6)\end{array}$ & $\begin{aligned} 83 & (90.2) \\
2 & (2.2) \\
6 & (6.5) \\
1 & (1.1)\end{aligned}$ & 0.52 \\
\hline $\begin{array}{l}\text { Anastomotic configuration, } \mathrm{n}(\%) \\
\text { EEA } \\
\text { SSA }\end{array}$ & $\begin{array}{l}50(82.0) \\
11(18.0)\end{array}$ & $\begin{array}{l}78(84.8) \\
14(15.2)\end{array}$ & 0.65 \\
\hline $\begin{array}{l}\text { Medication, n (\%) } \\
\text { 5-ASA } \\
\text { IM } \\
\text { Steroids }\end{array}$ & $\begin{array}{c}4(6.6) \\
14(23.0) \\
8(13.1)\end{array}$ & $\begin{array}{c}8(8.7) \\
21(22.8) \\
28(30.4)\end{array}$ & $\begin{array}{c}0.76 \\
0.99 \\
0.013\end{array}$ \\
\hline
\end{tabular}

${ }^{5}$ World Health Organization classification; mild anemia when serum hemoglobin (Hb) 11-12 g/dL for females and 11-13 g/dL for males, moderate for Hb 8-11 g/dL and severe for $\mathrm{Hb}<8 \mathrm{~g} / \mathrm{dL}$ (16)

IQR, interquartile range; TNF, tumor necrosis factor; BMI, body mass index; ASA, American Society of Anesthesiologists; ICR, ileocolic resection; EEA, end-to-end anastomosis; SSA, side-to-side anastomosis; 5-ASA, 5-aminosalicylic acid; IM, immunomodulators 
retrospective studies are conflicting. Some are in accordance with our findings, concluding that preoperative anti-TNFs significantly correlate with POC [22-24]. A multicenter retrospective study with 231 patients found that overall complications, anastomotic leak and IASC rates were not

Table 3 Postoperative complications

\begin{tabular}{lc}
\hline Complications & $\mathrm{n}(\%)$ \\
\hline Overall & $35(22.9)$ \\
IASC & $19(12.4)$ \\
Anastomotic leak & $18(11.8)$ \\
Intra-abdominal & $1(0.7)$ \\
collection/abscess & \\
Other & \\
Postoperative ileus & $6(3.9)$ \\
Hemorrhage & $4(2.6)$ \\
Unexplained fever & $4(2.6)$ \\
Wound infection & $3(2.0)$ \\
UTI & $2(1.3)$ \\
RTI & $1(0.7)$ \\
Deaths & $0(0.0)$ \\
Reoperation & $22(14.4)$ \\
\hline IASC, intra-abdominal septic complication; UTI, urinary tract infection; RTI, \\
respiratory tract infection
\end{tabular}

increased in patients under infliximab or adalimumab [17]. Furthermore, a nationwide prospective study from the REMIND group assessed the impact of anti-TNF agents by evaluating the preoperative serum trough levels; it was found that POC were not increased, regardless of the interval from last dosing or the serum levels before surgery [15]. Similarly, recently published data from another multicenter prospective cohort (PUCCINI trial) with 955 IBD patients, although not separating patients with $\mathrm{CD}$ from those with ulcerative colitis (UC), showed that anti-TNF medication within 3 months preoperatively was not an independent predictor for infectious complications, even when there were detectable serum drug levels at the time of surgery [25].

On the other hand, many studies have documented an increased risk for postoperative morbidity, especially septic POC [26,27]. Recent retrospective data from Leuven and Amsterdam, concerning 538 patients who had undergone ileocecal resection, reported an OR of 3.30 (95\%CI 1.099.99, $\mathrm{P}=0.035$ ) for anastomotic leakage for patients receiving anti-TNF medication [19]. In 2018, a prospective study of a nationwide cohort of 592 patients from France was published, in which anti-TNF within 3 months before ICR was an independent risk factor for overall POC [28]. Similarly, Kulaylat et al analyzed a large database of 2364 patients, adjusting for confounders such as emergency surgery, and concluded that
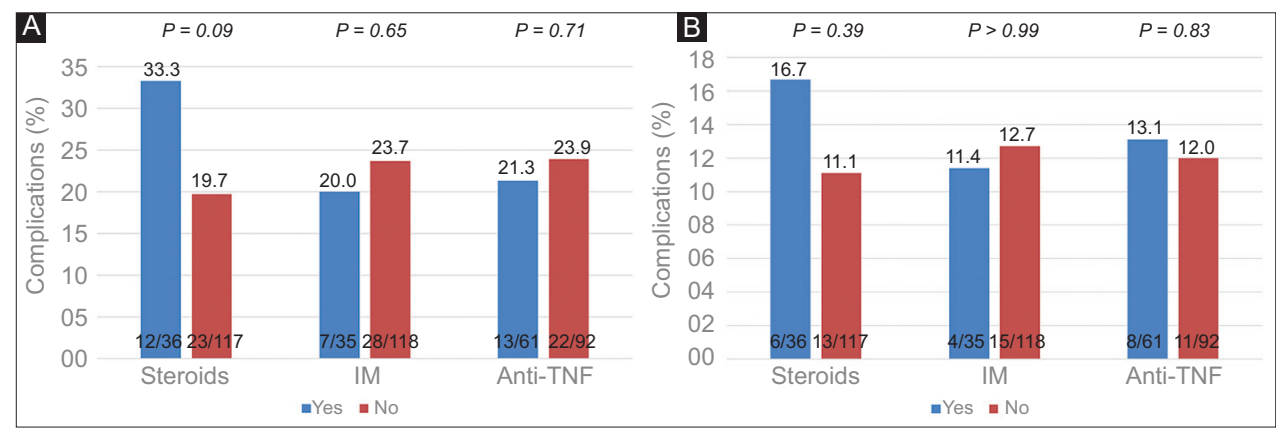

Figure 1 Univariate analysis for the correlation of overall (A) and intra-abdominal septic (B) postoperative complications with preoperative medication IM, immunomodulators; TNF, tumor necrosis factor
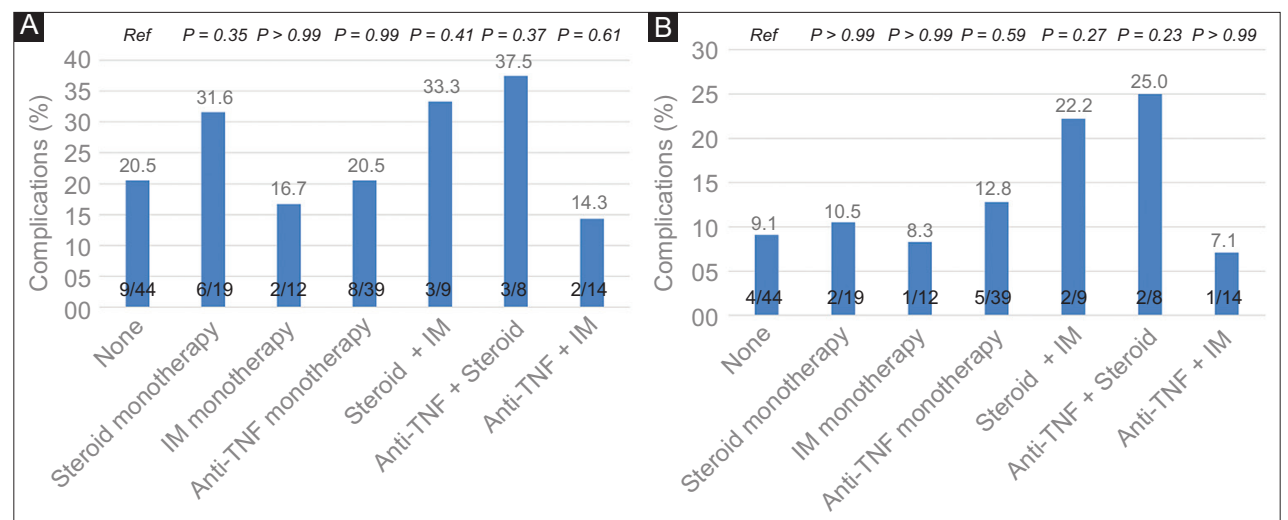

Figure 2 Univariate analysis for the correlation of overall (A) and intra-abdominal septic (B) postoperative complications with preoperative therapeutic regimen. All $\mathrm{P}$-values are $v$ s. none. Overall $\mathrm{P}=0.75$ (A) and 0.80 (B)

IM, immunomodulators; TNF, tumor necrosis factor 
Table 4 Univariate analysis for detection of risk factors for postoperative complications

\begin{tabular}{|c|c|c|c|c|c|}
\hline Risk factors & $\mathrm{n}$ & Overall complications $(\mathrm{n}=35)$ & P-value & IASC $(n=19)$ & P-value \\
\hline Age (years), median (IQR) & 153 & $39(28-48)$ vs 35 (28-46) & 0.43 & $\begin{array}{c}40(35-48) \text { vs } \\
35(28-47)\end{array}$ & 0.34 \\
\hline $\begin{array}{l}\text { Male sex, n (\%) } \\
\text { Yes } \\
\text { No }\end{array}$ & $\begin{array}{l}86 \\
67\end{array}$ & $\begin{array}{l}19(22.1) \\
16(23.9)\end{array}$ & 0.79 & $\begin{array}{c}9(10.5) \\
10(14.9)\end{array}$ & 0.41 \\
\hline $\begin{array}{l}\text { Smoking, n (\%) } \\
\text { Yes } \\
\text { No }\end{array}$ & $\begin{array}{l}61 \\
92\end{array}$ & $\begin{array}{l}17(27.9) \\
18(19.6)\end{array}$ & 0.23 & $\begin{array}{c}10(16.4) \\
9(9.8)\end{array}$ & 0.23 \\
\hline $\begin{array}{l}\text { BMI }\left(\mathrm{kg} / \mathrm{m}^{2}\right) \text {, mean } \pm \text { SD } \\
\quad<18.5, \mathrm{n}(\%) \\
18.5-25, \mathrm{n}(\%) \\
25-30, \mathrm{n}(\%) \\
30-35, \mathrm{n}(\%) \\
>35, \mathrm{n}(\%)\end{array}$ & $\begin{array}{r}16 \\
89 \\
37 \\
4 \\
1\end{array}$ & $\begin{array}{l}4(25.0) \\
22(24.7) \\
8(21.6) \\
0(0.0) \\
0(0.0)\end{array}$ & 0.79 & $\begin{array}{c}3(18.8) \\
12(13.5) \\
3(8.1) \\
0(0.0) \\
0(0.0)\end{array}$ & 0.73 \\
\hline $\begin{array}{l}\text { Anemia, } \mathrm{n}(\%)^{\varsigma} \\
\text { Yes } \\
\text { No }\end{array}$ & $\begin{array}{l}39 \\
65\end{array}$ & $\begin{array}{c}8(20.5) \\
13(20.0)\end{array}$ & 0.95 & $\begin{array}{c}3(7.7) \\
7(10.8)\end{array}$ & 0.74 \\
\hline $\begin{array}{l}\text { Albumin }>3.0 \mathrm{~g} / \mathrm{dL}, \mathrm{n}(\%) \\
>3.0, \mathrm{n}(\%) \\
<3.0, \mathrm{n}(\%)\end{array}$ & $\begin{array}{l}90 \\
14\end{array}$ & $\begin{array}{c}17(18.9) \\
4(28.6)\end{array}$ & 0.47 & $\begin{array}{l}8(8.9) \\
2(14.3)\end{array}$ & 0.62 \\
\hline $\begin{array}{l}\text { ASA Score, n (\%) } \\
\text { I/II } \\
\text { III - IV }\end{array}$ & $\begin{array}{c}143 \\
10\end{array}$ & $\begin{array}{c}32(22.4) \\
3(30.0)\end{array}$ & 0.70 & $\begin{array}{l}17(11.9) \\
2(20.0)\end{array}$ & 0.36 \\
\hline $\begin{array}{l}\text { Montreal - Age, n (\%) } \\
\text { A1 } \\
\text { A2 } \\
\text { A3 }\end{array}$ & $\begin{array}{c}23 \\
101 \\
29\end{array}$ & $\begin{array}{c}6(26.1) \\
25(24.8) \\
4(13.8)\end{array}$ & 0.43 & $\begin{array}{c}5(21.7) \\
13(12.9) \\
1(3.4)\end{array}$ & 0.14 \\
\hline $\begin{array}{l}\text { Montreal - Location, n (\%) } \\
\text { L1 } \\
\text { L2 } \\
\text { L3 }\end{array}$ & $\begin{array}{c}92 \\
9 \\
52\end{array}$ & $\begin{array}{l}21(22.8) \\
3(33.3) \\
11(21.2)\end{array}$ & 0.72 & $\begin{array}{c}12(13.0) \\
2(22.2) \\
5(9.6)\end{array}$ & 0.55 \\
\hline $\begin{array}{l}\text { Montreal - L4, n (\%) } \\
\text { Yes } \\
\text { No }\end{array}$ & $\begin{array}{c}4 \\
149\end{array}$ & $\begin{array}{c}0(0.0) \\
35(23.5)\end{array}$ & 0.57 & $\begin{array}{c}0(0.0) \\
19(12.8)\end{array}$ & $>0.99$ \\
\hline $\begin{array}{l}\text { Montreal - Behavior, n (\%) } \\
\text { B1 } \\
\text { B2 } \\
\text { B3 }\end{array}$ & $\begin{array}{c}9 \\
95 \\
49\end{array}$ & $\begin{array}{l}3(33.3) \\
21(22.1) \\
11(22.4)\end{array}$ & 0.74 & $\begin{array}{c}1(11.1) \\
12(12.6) \\
6(12.2)\end{array}$ & 0.99 \\
\hline $\begin{array}{l}\text { Perianal disease, } \mathrm{n}(\%) \\
\text { Yes } \\
\text { No }\end{array}$ & $\begin{array}{c}35 \\
118\end{array}$ & $\begin{array}{l}13(37.1) \\
22(18.6)\end{array}$ & 0.022 & $\begin{array}{l}9(25.7) \\
10(8.5)\end{array}$ & 0.016 \\
\hline $\begin{array}{l}\text { Disease duration (years), n (\%) } \\
<5 \\
5-10 \\
>10\end{array}$ & $\begin{array}{l}62 \\
36 \\
55\end{array}$ & $\begin{array}{l}10(16.1) \\
5(13.9) \\
20(36.4)\end{array}$ & $0.012^{\S \varsigma}$ & $\begin{array}{c}4(6.5) \\
3(8.3) \\
12(21.8)\end{array}$ & $0.029^{\varsigma s 5}$ \\
\hline $\begin{array}{l}\text { Extraintestinal manifestations, } \\
\mathrm{n}(\%) \\
\text { Yes } \\
\text { No }\end{array}$ & $\begin{array}{c}48 \\
105\end{array}$ & $\begin{array}{l}11(22.9) \\
24(22.9)\end{array}$ & 0.99 & $\begin{array}{l}9(18.8) \\
10(9.5)\end{array}$ & 0.11 \\
\hline $\begin{array}{l}\text { Previous resection, n (\%) } \\
\text { Yes } \\
\text { No }\end{array}$ & $\begin{array}{c}24 \\
129\end{array}$ & $\begin{array}{l}13(54.2) \\
22(17.1)\end{array}$ & $<0.001$ & $\begin{array}{l}9(37.5) \\
10(7.8)\end{array}$ & $<0.001$ \\
\hline
\end{tabular}


Table 4 (Continued)

\begin{tabular}{|c|c|c|c|c|c|}
\hline Risk factors & $\mathrm{n}$ & Overall complications $(n=35)$ & P-value & IASC $(n=19)$ & P-value \\
\hline Laparoscopic approach, n (\%) & & & 0.57 & & 0.71 \\
\hline Yes & 19 & $3(15.8)$ & & $3(15.8)$ & \\
\hline No & 134 & $32(23.9)$ & & $16(11.9)$ & \\
\hline Procedure, $n(\%)$ & & & 0.78 & & 0.88 \\
\hline ICR & 133 & $30(22.6)$ & & $16(12.0)$ & \\
\hline ICR + Strictureplasty & 5 & $1(20.0)$ & & $1(20.0)$ & \\
\hline $\mathrm{ICR}+$ resection & 13 & $4(30.8)$ & & $2(15.4)$ & \\
\hline ICR + resection + strictureplasty & 2 & $0(0.0)$ & & $0(0.0)$ & \\
\hline Anastomotic configuration, $\mathrm{n}(\%)$ & & & 0.88 & & 0.32 \\
\hline EEA & 128 & $29(22.7)$ & & $18(14.1)$ & \\
\hline SSA & 25 & $6(24.0)$ & & $1(4.0)$ & \\
\hline
\end{tabular}

${ }^{5}$ World Health Organization classification; anemia is defined when serum hemoglobin $<12 \mathrm{~g} / \mathrm{dL}$ for females and $<13 \mathrm{~g} / \mathrm{dL}$ for males $(16)$

${ }^{s 5}$ Post hoc analysis: $<5$ years vs. 5 - 10 years, $\mathrm{P}=0.77 ;<5$ years vs. $>10$ years, $\mathrm{P}=0.012 ; 5-10$ years vs. $>10$ years, $\mathrm{P}=0.019$. $\mathrm{P}$-values of $<0.017$ were considered statistically significant according to the Bonferroni correction

${ }^{\$ s \varsigma}$ Post hoc analysis: $<5$ years vs. $5-10$ years, $\mathrm{P}=0.71 ;<5$ years vs. $>10$ years, $\mathrm{P}=0.016 ; 5-10$ years vs. $>10$ years, $\mathrm{P}=0.09$. $\mathrm{P}$-values of $<0.017$ were considered statistically significant according to the Bonferroni correction

IASC, intra-abdominal septic complications; IQR, interquartile range; TNF, tumor necrosis factor; BMI, body mass index; ASA, American Society of Anesthesiologists; ICR, ileocolic resection; EEA, end-to-end anastomosis; SSA, side-to-side anastomosis; 5-ASA, 5-aminosalicylic acid; IM, immunomodulators

Table 5 Multivariate analysis for possible risk factors

\begin{tabular}{|c|c|c|c|c|}
\hline Risk factors & Overall complications Odds ratio $(95 \% \mathrm{CI})$ & P-value & IASC Odds ratio $(95 \% \mathrm{CI})$ & P-value \\
\hline \multicolumn{5}{|l|}{ Smoking } \\
\hline No & Ref & & Ref & \\
\hline Yes & $1.44(0.61-3.39)$ & 0.40 & $1.44(0.60-3.47)$ & 0.41 \\
\hline \multicolumn{5}{|c|}{ Montreal - Age } \\
\hline A1 & - & & Ref & \\
\hline $\mathrm{A} 2$ & - & & $1.01(0.29-3.49)$ & 0.99 \\
\hline $\mathrm{A} 3$ & - & & $0.45(0.08-2.40)$ & 0.35 \\
\hline \multicolumn{5}{|c|}{ Perianal disease } \\
\hline No & Ref & & Ref & \\
\hline Yes & $1.95(0.74-5.16)$ & 0.18 & $1.79(0.66-4.88)$ & 0.26 \\
\hline \multicolumn{5}{|c|}{ Disease duration (years) } \\
\hline$<5$ & Ref & & Ref & \\
\hline $5-10$ & $0.93(0.28-3.11)$ & 0.91 & $0.82(0.24-2.86)$ & 0.76 \\
\hline$>10$ & $1.94(0.74-5.11)$ & 0.18 & $1.76(0.65-4.77)$ & 0.27 \\
\hline \multicolumn{5}{|c|}{ Extraintestinal manifestations } \\
\hline No & - & & Ref & \\
\hline Yes & - & & $0.72(0.28-1.82)$ & 0.48 \\
\hline \multicolumn{5}{|c|}{ Previous resection } \\
\hline No & Ref & & Ref & \\
\hline Yes & $3.90(1.38-11.06)$ & 0.010 & $4.56(1.51-13.77)$ & 0.007 \\
\hline \multicolumn{5}{|l|}{ Steroids } \\
\hline No & Ref & & Ref & \\
\hline Yes & $1.99(0.78-5.13)$ & 0.15 & $1.91(0.72-5.03)$ & 0.19 \\
\hline \multicolumn{5}{|l|}{ IM } \\
\hline No & Ref & & Ref & \\
\hline Yes & $0.78(0.28-2.19)$ & 0.64 & $0.72(0.25-2.03)$ & 0.53 \\
\hline \multicolumn{5}{|l|}{ Anti-TNF } \\
\hline No & Ref & & Ref & \\
\hline Yes & $0.72(0.29-1.81)$ & 0.49 & $0.64(0.25-1.68)$ & 0.37 \\
\hline
\end{tabular}

CI, confidence interval; IASC, intra-abdominal septic complications; IM, immunomodulators; TNF, tumor necrosis factor 
the overall and septic POC are significantly higher in anti-TNF exposed patients [18].

A critical question is whether a surgeon should postpone an elective surgery according to the timing of the last drug infusion. Since evidence for the safety of anti-TNFs is conflicting, our practice was to perform elective ICRs when a period of at least 4 weeks had elapsed since the last administration of anti-TNF; we could assume that this time interval seems to be safe. A Danish nationwide cohort with 2293 CD patients concluded that anti-TNF administration within 14 days before surgery was not associated with a higher risk for POC compared to longer intervals [22]. A meta-analysis conducted for the comparison of different time intervals (within 4, 8 and 12 weeks) also found no significant differences [29]. Lau et al, in contrast to the PUCCINI trial and the prospective cohort from the REMIND group, found that anti-TNF serum levels at surgery of $\geq 3 \mu \mathrm{g} / \mathrm{mL}$ correlated with increased overall postoperative morbidity and infectious complications, while levels of $\geq 8 \mu \mathrm{g} / \mathrm{mL}$ correlated with overall postoperative morbidity and readmissions [30]. Nevertheless, in this study a major limitation was that data regarding the interval between last infusion and surgery were not obtained and therefore the optimal timing for last dosing could not be estimated. Overall, it seems that the preoperative serum levels of the drug do not help discriminate patients at risk and a safe time interval from last administration to surgery cannot be defined.

To date, since the conduction of well-designed randomized controlled trials is an unmet need because of ethical considerations, the literature provides clinicians with conflicting evidence about the perioperative safety of anti-TNF agents. This heterogeneity is reflected in the discrepancy of results from several meta-analyses that used the same pool of data. An interesting meta-analysis in 2013, by El-Hussuna et al, found a 1.6-fold increase in relative risk for anastomotic complications only when the analysis included studies with a low risk of bias [31]. Yang et al found an increased risk for overall and infectious complications in their meta-analysis and stated that the most appropriate definition with lower heterogeneity among studies would be infectious and non-infectious POC [32]. As for IASC, Huang in 2014 found that steroid use, hypoalbuminemia, previous surgery and preoperative abscess were predictors, but anti-TNF treatment was not [33]. In a 2018 meta-analysis with separate analysis for every category of POC described in previous studies (overall, major/minor, septic/nonseptic, surgical/medical) anti-TNFs were not associated with any kind of POC [34], whereas a more recent study in 2019, which included 7115 patients, concluded that there is a higher risk for infectious POC [35]. The European Crohn's and Colitis Organization published in 2020 the updated guidelines for CD, stating that current evidence does not support any correlation of anti-TNF agents with POC after abdominal surgery [36].

In the present study, none of the other medications had a significant impact on POC. However, we detected a trend for increased overall POC in patients under steroid treatment, with borderline significance $(\mathrm{P}=0.09)$. Nevertheless, this finding is biased by the fact that patients under high doses of steroids for a long period and who needed ICR were protected by a diverting ileostomy and were thus excluded from this cohort. The dose-related association of steroids with POC is definite [7,37]. Moreover, we tried to evaluate the effect of combined immunosuppressive therapy, but statistical significance was not reached for any possible combination of anti-TNFs, steroids and immunomodulators. However, there was a trend for increased overall POC and IASC when steroids were added to any other class of medication (Fig. 2). In accordance with our results, the REMIND Group study documented no association of anti-TNFs in combination with other immunosuppressants [15]. A recent large retrospective study found that double immunosuppression of steroids and biologicals was an independent risk factor for IASC [38]. Furthermore, McKenna's cohort in 2018 recorded an increased risk for IASC only with triple immunosuppression [16]. The 2 last studies included anti-TNFs as well as vedolizumab and ustekinumab as the "biological part" of the combined regimen; therefore, they could not conclude if a specific biological class, when combined, increased the risk. In our cohort, no patient was under a triple immunosuppressive regimen.

A history of previous resections was the only independent risk factor for overall POC and IASC in this cohort. The correlation of repeated resections with postoperative morbidity is not well documented in the literature. A multicenter retrospective study was the first to report this variable as an independent risk factor, but only for anastomotic leakage and not for IASC [17]. A subsequent single-center study by Johnston et al tried to confirm this finding and, although statistical significance was reached in univariate analysis, multivariate analysis was not conducted because of the limited data [39]. Our results are in accordance with 2 large, recently published, retrospective studies by the same team in the Mayo Clinic that documented previous resections as an independent risk factor for IASC $[16,38]$. Interestingly, this finding was confirmed by Huang's abovementioned meta-analysis in 2015, although none of the included studies reported previous surgery as a risk factor for IASC [33]. We therefore believe that our findings increase the evidence for the negative effect of previous resections on the postoperative course of $\mathrm{CD}$ patients. We speculate that this association could be attributed to the technical difficulties encountered in a previously operated abdomen, which could compromise the blood supply in bowel cut edges, in combination with the aggressive disease pattern in patients who require reoperations; both could impair anastomotic healing.

In the present cohort, patients were operated in the elective setting and were therefore adequately optimized with proper anemia and malnutrition management. The mean $\mathrm{Hb}$ concentration was $12.7 \mathrm{~g} / \mathrm{dL}$, with no difference between the "anti-TNF" and "no anti-TNF" groups, whereas no patient had severe anemia. Similarly, $86.5 \%$ of patients had serum albumin $>3 \mathrm{~g} / \mathrm{dL}$ and $89.1 \%$ of them BMI $>18.5 \mathrm{~kg} / \mathrm{m}^{2}$. Thus, anemia and malnutrition were not confirmed as established risk factors $[6,20,21]$. Anastomotic technique is a controversial risk factor in the literature; current evidence, although weak, supports the superiority of a stapled SSA configuration over the EEA in terms of lower risk for anastomotic leak [36]. In our study, EEA was the prevailing configuration, all anastomoses were hand-sewn and differences between EEA and SSA were not confirmed for either overall POC or IASC. Last, the penetrating-B3 phenotype as a predictor was also not 
confirmed [20,33]; patients with severe intra-abdominal sepsis and abscesses were either drained percutaneously or diverted.

This study has certain limitations that should be highlighted. First, the retrospective, single-center design and the consequent selection bias is a flaw. Furthermore, our population was not completely homogenous between anti-TNF and non-antiTNF patients in terms of age, disease duration and steroid medication. Second, adjustment for certain confounders, such as disease activity, was not feasible. Although inclusion criteria were selected in order to include CD patients with mild disease, non-septic and under proper optimization strategy, disease severity data with validated scores might be useful. Last, although a (probably arbitrary) minimum interval of 4 weeks before elective surgery was kept as a rule of thumb, precise data could not be obtained regarding the last administration of anti-TNFs in order to include it as a variable in our analysis. However, we believe that this study adds more evidence in the evolving problem of $\mathrm{POC}$ in CD patients.

In conclusion, in the present study, previous resection was an independent risk factor for overall POC and IASC after elective ileocolectomy, adding more evidence about this variable to the existing literature. Preoperative medications, focusing especially on patients exposed to anti-TNF drugs, were not associated with postoperative morbidity, either as monotherapy regimens or as immunosuppressive combination treatments. Thus, we believe that a surgeon should take into account all possible risk factors for POC and should try to optimize the patient, rather than postpone an elective resection considering only the anti-TNF therapy status.

\section{Summary Box}

\section{What is already known:}

- The rates of postoperative complications (POC) and intra-abdominal septic complications (IASC) after ileocolic resection (ICR) for Crohn's disease are high

- The impact of anti-tumor necrosis factor (TNF)- $\alpha$ agents on POC and IASC remains debatable

- Highly consistent risk factors for POC are chronic, high-dose steroid therapy, poor nutritional status, anemia, complicated disease, and surgery in the emergency setting

\section{What the new findings are:}

- Preoperative administration of anti-TNF agents, either as monotherapy or in combination with other immunosuppressants, did not correlate with the total POC or IASC in patients undergoing elective ICR with primary anastomosis

- The history of previous intestinal resection was associated with significantly higher rates of total POC and IASC

\section{References}

1. Torres J, Mehandru S, Colombel JF, Peyrin-Biroulet L. Crohn's disease. Lancet 2017;389:1741-1755.

2. Adegbola SO, Sahnan K, Warusavitarne J, Hart A, Tozer P. AntiTNF therapy in Crohn's disease. Int J Mol Sci 2018;19:2244.

3. Frolkis AD, Dykeman J, Negrón ME, et al. Risk of surgery for inflammatory bowel diseases has decreased over time: a systematic review and meta-analysis of population-based studies. Gastroenterology 2013;145:996-1006.

4. Biroulet LP, Loftus EV, Harmsen WS, et al. 490 Postoperative complications in a population-based Cohort of Crohn's disease. Gastroenterology 2010;138:S-70.

5. Papaconstantinou I, Zeglinas C, Gazouli M, et al. The impact of peri-operative anti-TNF treatment on anastomosis-related complications in Crohn's disease patients. A critical review. J Gastrointest Surg 2014;18:1216-1224.

6. Patel KV, Darakhshan AA, Griffin N, Williams AB, Sanderson JD, Irving PM. Patient optimization for surgery relating to Crohn's disease. Nat Rev Gastroenterol Hepatol 2016;13:707-719.

7. Nguyen GC, Elnahas A, Jackson TD. The impact of preoperative steroid use on short-term outcomes following surgery for inflammatory bowel disease. J Crohns Colitis 2014;8:1661-1667.

8. Ahmed Ali U, Martin ST, Rao AD, Kiran RP. Impact of preoperative immunosuppressive agents on postoperative outcomes in Crohn's disease. Dis Colon Rectum 2014;57:663-674.

9. McKenna NP, Lightner AL. Preoperative considerations in inflammatory bowel disease. Surg Clin North Am 2019;99:1083-1094.

10. Williams EP, Mesidor M, Winters K, Dubbert PM, Wyatt SB. Overweight and obesity: prevalence, consequences, and causes of a growing public health problem. Curr Obes Rep 2015;4:363-370.

11. Haynes SR, Lawler PG. An assessment of the consistency of ASA physical status classification allocation. Anaesthesia 1995;50:195-199.

12. Silverberg MS, Satsangi J, Ahmad T, et al. Toward an integrated clinical, molecular and serological classification of inflammatory bowel disease: report of a Working Party of the 2005 Montreal World Congress of Gastroenterology. Can J Gastroenterol 2005;19 Suppl A:5A-36A.

13. Weimann A, Braga M, Carli F, et al. ESPEN guideline: Clinical nutrition in surgery. Clin Nutr 2017;36:623-650.

14. WHO. Haemoglobin concentrations for the diagnosis of anaemia and assessment of severity. Geneva: World Health Organization; 2011. Available from: https://www.who.int/vmnis/indicators/ haemoglobin.pdf [Accessed 20 July 2020].

15. Fumery M, Seksik P, Auzolle C, et al; REMIND study group investigators. Postoperative complications after ileocecal resection in Crohn's disease: a prospective study from the REMIND group. Am J Gastroenterol 2017;1 12:337-345.

16. McKenna NP, Habermann EB, Glasgow AE, Dozois EJ, Lightner AL. Intra-abdominal sepsis after ileocolic resection in Crohn's disease: the role of combination immunosuppression. Dis Colon Rectum 2018;61:1393-1402.

17. Yamamoto T, Spinelli A, Suzuki Y, et al. Risk factors for complications after ileocolonic resection for Crohn's disease with a major focus on the impact of preoperative immunosuppressive and biologic therapy: A retrospective international multicentre study. United European Gastroenterol J 2016;4:784-793.

18. Kulaylat AN, Kulaylat AS, Schaefer EW, et al. The impact of preoperative anti-TNFalpha therapy on postoperative outcomes following ileocolectomy in Crohn's disease. J Gastrointest Surg 2020 Jan 21 [Online ahead of print]. doi: 10.1007/s11605-019-04334-6

19. de Buck van Overstraeten A, Eshuis EJ, Vermeire S, et al. Shortand medium-term outcomes following primary ileocaecal resection for Crohn's disease in two specialist centres. Br J Surg 2017;104:1713-1722. 
20. Alves A, Panis Y, Bouhnik Y, Pocard M, Vicaut E, Valleur P. Risk factors for intra-abdominal septic complications after a first ileocecal resection for Crohn's disease: a multivariate analysis in 161 consecutive patients. Dis Colon Rectum 2007;50:331-336.

21. Morar PS, Hodgkinson JD, Thalayasingam S, et al. Determining predictors for intra-abdominal septic complications following ileocolonic resection for Crohn's disease-considerations in preoperative and peri-operative optimisation techniques to improve outcome. J Crohns Colitis 2015;9:483-491.

22. Nørgård BM, Nielsen J, Qvist N, Gradel KO, de Muckadell OB, Kjeldsen J. Pre-operative use of anti-TNF- $\alpha$ agents and the risk of post-operative complications in patients with Crohn's disease-a nationwide cohort study. Aliment Pharmacol Ther 2013;37:214-224.

23. Myrelid P, Marti-Gallostra M, Ashraf S, et al. Complications in surgery for Crohn's disease after preoperative antitumour necrosis factor therapy. Br J Surg 2014;101:539-545.

24. Kotze PG, Saab MP, Saab B, et al. Tumor necrosis factor alpha inhibitors did not influence postoperative morbidity after elective surgical resections in Crohn's disease. Dig Dis Sci 2017;62:456-464.

25. Cohen BL, Fleshner P, Kane SV, et al. Anti-tumor necrosis factor therapy is not associated with post-operative infection: results from prospective cohort of ulcerative colitis and Crohn's disease patients undergoing surgery to identify risk factors for postoperative infection I (Puccini). Gastroenterology 2019;156:S-80.

26. Syed A, Cross RK, Flasar MH. Anti-tumor necrosis factor therapy is associated with infections after abdominal surgery in Crohn's disease patients. Am J Gastroenterol 2013;108:583-593.

27. Serradori T, Germain A, Scherrer ML, et al. The effect of immune therapy on surgical site infection following Crohn's Disease resection. Br J Surg 2013;100:1089-1093.

28. Brouquet A, Maggiori L, Zerbib P, et al; GETAID chirurgie group. Anti-TNF therapy is associated with an increased risk of postoperative morbidity after surgery for ileocolonic Crohn disease: results of a prospective nationwide cohort. Ann Surg 2018;267:221-228.

29. Qiu Y, Zheng Z, Liu G, Zhao X, He A. Effects of preoperative antitumour necrosis factor alpha infusion timing on postoperative surgical site infection in inflammatory bowel disease: A systematic review and meta-analysis. United European Gastroenterol J 2019;7:1198-1214.

30. Lau C, Dubinsky M, Melmed G, et al. The impact of preoperative serum anti-TNF $\alpha$ therapy levels on early postoperative outcomes in inflammatory bowel disease surgery. Ann Surg 2015;261:487-496.

31. El-Hussuna A, Krag A, Olaison G, Bendtsen F, Gluud LL. The effect of anti-tumor necrosis factor alpha agents on postoperative anastomotic complications in Crohn's disease: a systematic review. Dis Colon Rectum 2013;56:1423-1433.

32. Yang ZP, Hong L, Wu Q, Wu KC, Fan DM. Preoperative infliximab use and postoperative complications in Crohn's disease: a systematic review and meta-analysis. Int J Surg 2014;12:224-230.

33. Huang W, Tang Y, Nong L, Sun Y. Risk factors for postoperative intra-abdominal septic complications after surgery in Crohn's disease: A meta-analysis of observational studies. J Crohns Colitis 2015;9:293-301.

34. Xu Y, Yang L, An P, Zhou B, Liu G. Meta-analysis: the influence of preoperative infliximab use on postoperative complications of Crohn's disease. Inflamm Bowel Dis 2019;25:261-269.

35. Lin YS, Cheng SW, Wang YH, Chen KH, Fang CJ, Chen C. Systematic review with meta-analysis: risk of post-operative complications associated with pre-operative exposure to antitumour necrosis factor agents for Crohn's disease. Aliment Pharmacol Ther 2019;49:966-977.

36. Adamina $\mathrm{M}$, Bonovas $\mathrm{S}$, Raine $\mathrm{T}$, et al. ECCO guidelines on therapeutics in Crohn's disease: surgical treatment. J Crohns Colitis 2020;14:155-168.

37. Subramanian V, Saxena S, Kang JY, Pollok RC. Preoperative steroid use and risk of postoperative complications in patients with inflammatory bowel disease undergoing abdominal surgery. Am J Gastroenterol 2008;103:2373-2381.

38. Lightner AL, McKenna NP, Alsughayer A, et al. Biologics and 30-day postoperative complications after abdominal operations for Crohn's disease: are there differences in the safety profiles? Dis Colon Rectum 2019;62:1352-1362.

39. Johnston WF, Stafford C, Francone TD, et al. What is the risk of anastomotic leak after repeat intestinal resection in patients with Crohn's disease? Dis Colon Rectum 2017;60:1299-1306. 